\title{
Design e Avaliação de um Jogo Educacional Web Inclusivo Multidispositivo e Customizável seguindo o conjunto EduGameAccess-M
}

\author{
Joice Lee Otsuka ${ }^{1}$, Ana Paula de Sousa Reis ${ }^{2}$, Caio Vinicius B. Santos, \\ Jhonata Nícolas C. Querobim, Mariana Z. Sabino, Delano M. Beder ${ }^{1}$ \\ ${ }^{1}$ Departamento de Computação - Universidade Federal de São Carlos (UFSCar) \\ ${ }^{2}$ Instituto Federal do Sul de Minas Gerais - Campus Poços de Caldas \\ joice@ufscar.br,fisicaanapaula@gmail.com
}

\begin{abstract}
This article presents the Forca Inclusiva's design, implementation and evaluation process. Forca Inclusiva is an educational game with customizable content, designed to include visually impaired people. The design process was guided by the set of recommendations EduGameAccess-M, which integrates educational, gameplay and accessibility aspects for people with visual impairments in multi-device games. The main aim of the research was to investigate the effectiveness of the referred set of recommendations in the design and evaluation of an inclusive web educational game, in versions for desktop and mobile devices. The initial results indicate that the proposed set was effective in supporting the development of the aforementioned game. This work was developed in the scope of the research project: Jogos e a Inclusão na Educação (Edital de Inovação CAPES - 42/2017)
\end{abstract}

Resumo. Este artigo apresenta o processo de design, implementação e avaliação da Forca Inclusiva, um jogo educacional de conteúdo customizável, projetado para incluir pessoas com deficiência visual. O processo de design foi norteado pelo conjunto de recomendações EduGameAccess-M, que integra aspectos educacionais, de jogabilidade e de acessibilidade para pessoas com deficiência visual em jogos para diferentes dispositivos. O objetivo da pesquisa foi investigar a efetividade do referido conjunto de recomendações no design e avaliação de um jogo educacional web inclusivo, em versões para dispositivos desktop e móveis. Os resultados iniciais obtidos indicam que o conjunto foi efetivo no apoio ao desenvolvimento do jogo em questão. Este trabalho foi desenvolvido no escopo do projeto de pesquisa: Jogos e a Inclusão na Educação (Edital de Inovação CAPES - 42/2017).

\section{Introdução}

De acordo com o Relatório Mundial sobre a Deficiência, publicado pela Organização Mundial da Saúde [OMS, 2011], aproximadamente 1 bilhão de pessoas no mundo possuem algum tipo de deficiência. Dados do Censo 2010 [IBGE , 2010] revelam que $23.9 \%$ da população brasileira possuem algum tipo de deficiência. O relatório da OMS alerta que "Garantir que as crianças com deficiência recebam educação de boa qualidade num ambiente inclusivo deveria ser prioridade em todos os países".

Nesse contexto, o desenvolvimento de recursos educacionais deve ter a inclusão 
como um de seus requisitos. Assim, os jogos educacionais, assim como todos os recursos educacionais, deveriam ser projetados seguindo abordagens mais inclusivas, buscando possibilitar experiências compartilhadas e equivalentes entre pessoas com e sem deficiência. É importante destacar que para ser ser inclusivo não basta ser acessível, ou seja, o design inclusivo busca incluir pessoas com deficiência e não apenas prover uma solução que seja acessível a um determinado público. De acordo com Östblad et al. (2014), para um jogo ser inclusivo, o grupo a ser incluído deve ser considerado em todos os aspectos do jogo e a experiência de jogo deve ser tão similar quanto possível, independentemente do jogador.

Entretanto, infelizmente atualmente a maior parte dos jogos enfatizam informações visuais, sem prover alternativas de acesso a pessoas com determinados tipos de deficiência visual. Existem várias iniciativas de jogos acessíveis, em especial os audiogames [FRIBERG e GÄRDENFORS, 2004; FIZEK et al., 2015], mas estas soluções geralmente não promovem experiências inclusivas, pois são projetadas com foco apenas nas pessoas com deficiência visual.

Com o intuito de contribuir para o desenvolvimento de jogos educacionais inclusivos, foi proposto o conjunto de recomendações EduGameAccess, que integra aspectos educacionais, de jogabilidade e acessibilidade de pessoas com deficiência visual [FONTOURA JUNIOR 2018, NETO et al. 2019]. Esse conjunto foi ampliado para considerar também o design de jogos multidispositivos, em especial para desktop e mobile, dando origem ao conjunto EduGameAccess-M [REIS, 2020].

$\mathrm{O}$ objetivo da pesquisa foi investigar a efetividade do conjunto de recomendações EduGameAccess-M no design e avaliação de um jogo educacional web inclusivo, em versões para dispositivos desktop e móveis. Dessa forma, este artigo apresenta o design, a implementação e a avaliação da Forca Inclusiva, um jogo web inclusivo, customizável e multidispositivo, projetado seguindo o referido conjunto. Os problemas de design identificados a partir do uso das recomendações, bem como as soluções de design propostas são apresentados. São analisados os resultados obtidos em avaliações por inspeção por 4 especialistas e testes de jogabilidade conduzidos com 19 usuários: 5 cegos, 4 daltônicos, 5 com baixa visão e 5 sem deficiência visual.

As seções 2 e 3 apresentam a fundamentação teórica e trabalhos relacionados. $\mathrm{Na}$ sequência, as seções 4 a 6 apresentam as principais considerações sobre o design, a implementação e a avaliação do jogo Forca Inclusivo. Finalmente, na seção 7 são apresentadas as considerações finais.

\section{Fundamentação teórica}

A acessibilidade nos jogos pode ser definida como a facilidade de uso de um jogo, mesmo com condições limitantes, deficiências funcionais, permanentes ou temporárias. Para Archambault et al. (2007), a acessibilidade em jogos lida com um problema mais complexo do que a acessibilidade em outros tipos de software. Como os jogos permitem que os jogadores realizem ações significativas e vejam os resultados de suas decisões e escolhas, é importante que os jogos acessíveis mantenham seus aspectos lúdicos, sem perder a essência de serem objetos de diversão e entretenimento.

De acordo com Yuan et al (2011), a acessibilidade em jogos visa criar 
aplicativos com rica experiência de interação e de diferentes tipos de gêneros. A revisão bibliográfica realizada pelos autores sobre acessibilidade em jogos apresenta correlações entre deficiências e jogos, que trata dos principais gêneros, déficits e de diretrizes já definidas para solucionar esses problemas. Percebe-se também que, embora a maioria dos jogos busque considerar uma variedade de necessidades de interação diferentes, seu foco geralmente está em um único tipo de deficiência.

Grammenos, Savidis e Stephanidis (2009) propõem o design universal de jogos por meio de uma estrutura de design unificada baseada no design centrado no usuário. Nessa proposta, especialistas em avaliação de acessibilidade e usabilidade trabalham juntos durante o desenvolvimento do jogo com o intuito de melhorá-lo, fazendo alterações e aprimorando-o em qualquer momento.

Algumas recomendações de jogabilidade e acessibilidade em jogos estão disponíveis na literatura. Korhonen (2016) propõe um conjunto de 47 heurísticas de jogabilidade, organizadas em 5 grupos: usabilidade, gameplay, multiplayer, ciência ao contexto e mobilidade. Este conjunto é relevante para o presente estudo por abordar recomendações para o desenvolvimento de jogos para dispositivos móveis, porém, não abordam questões relacionadas à acessibilidade e inclusão. Um grupo composto por estúdios, especialistas e acadêmicos propôs as Game Accessibility Guidelines (GAG) [GAG, 2019], uma lista completa de diretrizes, divididas por deficiências e subdivididas em diretrizes básicas, intermediárias e avançadas, de acordo com a complexidade de sua conformidade e viabilidade do projeto. A International Game Developers Association (IGDA) desenvolveu um conjunto de recomendações de acessibilidade para jogos considerando diferentes tipos de deficiências. Entre essas, 16 recomendações são específicas para pessoas com deficiência visual [IGDA, 2019].

\section{Trabalhos relacionados}

O Audio Game Hub, um jogo para dispositivos móveis e computadores desktop, apresenta uma coleção de mini-jogos tradicionais acessíveis, que trazem interações através de instruções de áudio e efeitos de estéreo panorâmico, que permitem a localização do som em um plano bidimensional (esquerda, direita e centro), por meio do uso de fones de ouvido estereofônicos. Embora o jogo apresente uma interface gráfica minimalista para jogadores com visão, todas as interações nos menus e botões têm efeitos sonoros característicos e feedbacks sonoros que levam os jogadores cegos a navegar pelas opções disponíveis e acessar os mini-jogos [FIZEK et al, 2015].

Glinert (2008) apresenta um estudo de caso com o jogo AudiOdyssey, que teve como objetivo atender os usuários com deficiência visual, porém sem perder o interesse daqueles usuários sem deficiência. $\mathrm{O}$ jogo priorizou conciliar os atributos de jogabilidade e acessibilidade sendo que os desafios e níveis de dificuldade são compartilhados por todos que jogam. O AudiOdyssey foi produzido e avaliado por pesquisadores da Universidade de Singapura e estudantes do MIT.

Grabski et al. (2016) apresentam uma pesquisa que teve como objetivo a criação de um jogo acessível 3D competitivo para ser jogado por pessoas com e sem deficiência visual, com experiências equivalentes para todos. Os usuários sem deficiência visual tinham como recurso a comunicação visual, interação por meio de um kinect, enquanto os usuários com deficiência visual utilizavam a comunicação por toque 
IX Congresso Brasileiro de Informática na Educação (CBIE 2020)

Anais do XXXI Simpósio Brasileiro de Informática na Educação (SBIE 2020)

para interagir com o ambiente por haptics, e o feedback em forma de som 3D.

\section{Recomendações para o design de jogos educacionais inclusivos}

Nesta seção são apresentados brevemente dois conjuntos de recomendações definidos em trabalhos anteriores do grupo, com o intuito de contribuir para o desenvolvimento de jogos educacionais inclusivos.

\subsection{EduGameAccess}

O conjunto de recomendações EduGameAccess [FONTOURA JUNIOR, 2018] abrange aspectos de jogabilidade, acessibilidade e educacionais. $\mathrm{O}$ conjunto integra e refina três recomendações: (i) Game Accessibility Guidelines (GAG Game Accessibility Guidelines 2019); (ii) Play - as heurísticas do Desurvire para usabilidade em jogos [DESURVIRE e WIBERG, 2009] e (iii) princípios de aprendizado de Gee [GEE, 2007]. Como algumas dessas recomendações não se aplicam a jogos educacionais 2D, o EduGameAccess seleciona um subconjunto relevante de diretrizes considerando esse escopo, adapta e integra esse subconjunto. Além disso, como equipe de desenvolvimento de jogos educacionais geralmente possuem recursos escassos, esse conjunto é dividido em 3 níveis: básico (31 itens), intermediário (15) e avançado (7), de acordo com a relevância e o custo da implementação. Estudos anteriores do utilizando o EduGameAccess [NETO et al., 2019] mostram que o subconjunto básico é suficiente para orientar o design de um jogo educacional inclusivo 2D.

\subsection{EduGameAccess-M}

O conjunto EduGameAccess-M amplia o conjunto básico do EduGameAccess, com o intuito de contemplar o design de jogos inclusivos que possam ser utilizados em computadores desktop e dispositivos móveis. Neste novo conjunto, todas as 31 recomendações do conjunto básico foram revisadas para contemplar o design inclusivo para desktop e dispositivos móveis e 8 novas recomendações foram incluídas para abordar aspectos relacionados à mobilidade, com base no conjunto de recomendações de design de jogos móveis de Korhonen (2016) e resultados obtidos em estudos de caso envolvendo o desenvolvimento de jogos inclusivos no Laboratório de Objetos de Aprendizagem (LOA) da UFSCar [REIS, 2020]. O desenvolvimento do jogo Forca Inclusivo, apresentado nas próximas seções, foi um desses estudos de caso.

\section{Design do jogo Forca Inclusiva}

O jogo Forca Inclusiva foi projetado tendo como base uma versão web do tradicional jogo Forca, previamente desenvolvida no LOA. Ambas as versões foram criadas para serem integradas à plataforma REMAR, que provê um conjunto de ferramentas que facilitam a criação, publicação e acompanhamento de instâncias abertas de jogos educacionais, com conteúdo customizado diretamente pelo professor, criados a partir de modelos de jogos disponíveis na plataforma [BEDER e OTSUKA, 2019].

A versão web inclusiva foi projetada para atender os seguintes requisitos principais: (1) incluir pessoas com deficiência visual; (2) prover boa jogabilidade a esse público-alvo tanto em computadores desktop quanto em dispositivos móveis; (3) ser publicado como um modelo de jogo da plataforma REMAR. Para o design da versão 
inclusiva, primeiramente foi realizada uma avaliação do jogo original usando o conjunto EduGameAccess- $M$, com o intuito de identificar recomendações não atendidas. A avaliação foi realizada pela equipe de desenvolvimento do jogo, composta por 3 estudantes de graduação em Computação, sob supervisão da professora orientadora. A seguir são apresentados os principais problemas identificados e soluções propostas.

Tabela 1. Problemas identificados e soluções propostas
P: Fonte não legível em tamanho e estilo.
S: Fonte Another de 26px foi substituída pela fonte Tahoma 30px.

P: Falta de contraste entre texto e o plano de fundo: linhas azuis no fundo da tela, teclas opacas, texto sem fundo, fundo texturizado, escolha de cores dos texto com baixo contraste.

S: Fundo do jogo foi clareado juntamente com as linhas azuis, fundo inserido nos botões, cores de textos ajustados para melhor contraste.

P: Informações primordiais transmitidas apenas por cor: teclas selecionadas aparecem apenas em vermelho e indicação apenas visual das letras já selecionadas.

S: Teclas selecionadas mudam de estilo e quando selecionadas retornam feedback sonoro de que a letra já foi escolhida.

P: Sem controles de volume separados para efeitos, fala e música de fundo.

S: Implementação de controle de volume acessível e separado para música de fundo, leitura de tela/dublagens pré-gravadas e efeitos sonoros. Nas duas versões, o acesso às opções de controle de áudio pode ser acessada por meio do botão ÁUDIO na tela inicial do jogo. Na versão desktop ${ }^{l}$, podem ser acessadas também durante o jogo, por meio da tecla ESC. Na versão mobile ${ }^{2}$, podem ser acessadas por meio do botão OPÇÕES no canto superior esquerdo da tela.

P: Sem dublagem pré-gravada para o texto, menus e instaladores. Além disso, todas as questões do jogo são customizáveis e o áudio desse conteúdo só pode ser gerado a partir da customização. S: Na versão desktop, todo o conteúdo fixo do jogo foi gravado em estúdio. Para o conteúdo customizável, inicialmente foi utilizada a Javascript API SpeechSynthesisUtterance. Entretanto, o comportamento da API se mostrou instável e incompatível com algumas versões de navegadores e/ou sistemas operacionais. Dessa forma, optou-se por gerar todos os áudios no processo de customização, por meio de um sintetizador integrado à plataforma REMAR.

$\mathrm{Na}$ versão mobile, optou-se por utilizar o recurso de Leitura de Tela nativa do Android, considerando a familiaridade do público-alvo com essa solução, que além de prover uma boa leitura dos elementos da tela, também facilita a navegação e a seleção de elementos com duplo toque.

P: Sem feedbacks sonoros (apenas visual) para erros, acertos, status do enforcamento (vidas), letras escolhidas, letras na palavra em construção, palavra completa no fim da fase. Também não há leitura de botões de controle, créditos e instruções do jogo.

S: Áudios foram gravados para erros, acertos, status do enforcamento (número de vidas restantes), botões de controle, créditos e tutorial. Foram gravadas todas as letras do alfabeto para a inserção de feedback sonoro para cada letra selecionada e letras na palavra em construção. Também foi criado um efeito sonoro emitido quando se tenta selecionar uma letra indisponível (já selecionada anteriormente ).

P: Elementos interativos do jogo pequenos e pouco espaçados: tela não é bem aproveitada e

\footnotetext{
${ }^{1}$ Consiste do acesso ao jogo através de um navegador presente em um computador desktop.

${ }^{2}$ Consiste do acesso ao jogo através de um navegador presente em um dispositivo móvel Android.
} 
teclas são pequenas e não são bem espaçadas.

$\mathrm{S}$ : Layout revisado tanto na versão desktop quanto na versão mobile. Elementos espaçados, fontes e botões e teclado virtual aumentados. Foi necessário um redesign total do layout da versão mobile, que inicialmente era o mesmo da versão desktop. As principais alterações na versão mobile foram: (1) revisão do número de itens na tela, priorizando as informações essenciais para o jogo (número de vidas, dica, palavra em construção e teclado virtual); (2) botões de acesso rápido às opções de status e controle de áudio; (3) maior responsividade, para reajustar os componentes e textos na tela de acordo com o tamanho da tela do dispositivo.

P: Sem orientações para pessoas com ou sem deficiência visual sobre os principais controles do jogo.

S: Criação de instruções contendo o objetivo do jogo e principais controles, podendo ser acessada no menu inicial e dentro do jogo, a qualquer momento.

P: Os indicadores de status (número de vidas, letras já escolhidas, estado da palavra em construção) não disponíveis para acesso a qualquer momento por pessoas cegas ou baixa visão severa.

$\mathrm{S}$ : Na versão desktop foram adicionadas teclas de atalho para acesso direto à dica, ao número de vidas, ao status da palavra em construção, às letras já escolhidas e à pontuação do jogador. Na versão mobile foi adicionado um botão STATUS no canto superior direito da tela, dando acesso a um menu com acesso a essas informações.

P: Pontuação de difícil acesso para jogadores com deficiência visual

S: Informar a pontuação também por meio de áudio.

\section{Implementação da Forca Inclusiva}

O jogo foi implementado utilizando as tecnologias HTML/CSS/Javascript e encontra-se disponível para ser customizado na plataforma REMAR, na qual advoga-se que os jogos customizáveis sejam modulares, delimitando claramente os pontos de customização do jogo. Nesse sentido, cada ponto de customização deve estar vinculado a um arquivo distinto, presente no código-fonte do jogo e que pode ser substituído durante o processo de customização. Assim, os pontos de customização do jogo Forca Inclusiva são: (i) um arquivo JSON que contém as palavras e as dicas associadas; (ii) um conjunto de arquivos $\mathrm{mp} 3^{3}$, referentes aos áudios de cada palavra e cada dica do jogo e (iii) arquivo de imagem que pode ser utilizado para customização do plano de fundo.

Para a implementação das principais soluções de acessibilidade que foram adotadas, teve-se o cuidado de transmitir as informações essenciais da forma mais clara e objetiva possível. Assim para uma ação do jogador, como a de escolher uma letra, feedbacks sonoros são apresentados para indicar erros ou acertos. No caso de erros, o número de vidas restantes é informado, enquanto que no caso de acertos, o status atualizado da palavra a ser descoberta é informado. Além disso, um sistema de atalhos (teclas numéricas na versão desktop, e uma lista de botões na versão mobile) foi inserido para consulta dessas informações a qualquer momento durante o jogo. Outro aspecto importante trabalhado foi a navegabilidade, que mostrou-se um desafio principalmente na versão mobile. Na solução adotada procurou-se manter a consistência e

\footnotetext{
${ }^{3}$ Esses arquivos são gerados por um sintetizados integrado à plataforma REMAR.
} 
padronização, dispondo os elementos em posições padronizadas e intuitivas, no qual os jogadores acessam através do feedback do leitor de tela de seu dispositivo móvel. O código fonte do jogo está disponível no github do LOA/UFSCar' ${ }^{4}$.

\section{Avaliação da Forca Inclusiva}

A avaliação do jogo Forca inclusiva foi feita em duas etapas distintas: (1) avaliação por inspeção de especialistas com base no conjunto EduGameAccess-M, para verificar se as as recomendações foram atendidas por meio das soluções apresentadas na seção 4; (2) testes de jogabilidade realizados com quatro grupos de usuários: cegos, daltônicos, pessoas com baixa visão e sem deficiência visual. Todos os participantes receberam o Termo Consentimento Livre e Esclarecido (TCLE) ou, no caso de menores, o Termo de Assentimento Livre e Esclarecido (TALE), explicando o objetivo do estudo, etapas do estudo, riscos e benefícios. Também vale destacar que a pesquisa foi aprovada pelo Comitê de Ética em Pesquisa da UFSCar.

A avaliação por inspeção foi realizada por quatro especialistas: três com experiência de 3 a 7 anos em design e/ou avaliação de jogos educacionais e um com 3 anos de experiência em IHC. Os especialistas receberam o TCLE e um questionário online para a avaliação do jogo. Os avaliadores analisaram o grau de adequação do jogo a cada recomendação, usando uma escala Likert de 1 (discordo totalmente) a 7 (concordo totalmente) e comentários textuais opcionais. Os resultados obtidos mostraram que $75 \%$ das notas foram maiores ou iguais a 5 , o que demonstra a conformidade do jogo Forca Inclusiva ao conjunto EduGameAccess-M, ou seja, o redesign apresentado na seção 4 foi efetivo. Observou-se que as recomendações que receberam nota menor ou igual a 4 se referiam às recomendações pouco aplicáveis ao tipo de jogo avaliado, por exemplo, o jogo não possui fases. Além disso, observou-se que recomendações 13, 38 e 39 não se aplicam ao jogo Forca Inclusiva.

Os testes de jogabilidade foram conduzidos utilizando dois dispositivos: um smartphone e um computador desktop, de forma que todos os usuários pudessem testar o jogo em suas duas versões, verificando a interação e eventuais dificuldades encontradas em cada um dos dispositivos. Foram recrutados voluntários para 4 grupos de usuários: 5 pessoas cegas (4 do sexo masculino e 1 do sexo feminino, com idades entre 12 e 38 anos e escolaridade entre o ensino fundamental e o superior), 5 pessoas com baixa visão (todos adolescentes com idade média de 12 anos, familiarizados com o uso de smartphones e tablets), 4 daltônicos (todos do sexo masculino, com idade entre 21 e 35 anos, com ensino superior, familiarizados com uso de smartphones e desktops), 5 pessoas sem deficiência visual (com idade entre 14 e 17 anos, alunos de escola pública, familiarizados com ambos os dispositivos utilizados no teste). Os usuários cegos e os com baixa visão foram selecionados aleatoriamente dentre alunos matriculados em uma associação de assistência a deficientes visuais no interior do estado de Minas Gerais. Os usuários daltônicos foram recrutados por meio de contato em redes sociais e os usuários sem deficiência visual em uma escola estadual no interior do estado de Minas Gerais. Todos os usuários utilizaram seus próprios smartphones nos testes e os computadores desktop foram cedidos pelas instituições coparticipantes.

\footnotetext{
${ }^{4}$ https://github.com/LOA-SEAD
} 
Os usuários foram orientados a "pensar alto" (protocolo Think Aloud) durante a interação com o jogo, para que suas reações, dúvidas e sentimentos pudessem ser melhor identificados. Durante os testes foi observado que todos os usuários cegos mostraram ter facilidade com o uso do jogo nos dois dispositivos (mesmo os com menos experiência); todos os participantes de baixa visão tiveram facilidade em relação à mecânica do jogo e não utilizaram a lupa como recurso para o teste (um dos usuários com alta severidade, preferiu se guiar pelas informações sonoras); todos os participantes daltônicos utilizaram seus próprios aparelhos smartphones e realizaram o teste rapidamente; todos os participantes sem deficiência visual, estavam familiarizados com recursos tecnológicos e não tiveram dificuldades com a mecânica e interação no jogo. Todos entenderam bem os objetivos do teste e enfatizaram o desejo de que os jogos se tornassem parte de seus cotidianos escolares.

Após os testes de jogabilidade, os usuários foram convidados a responder um questionário baseado no instrumento de avaliação de jogos educacionais EGameFlow [FU et al. 2009], que utiliza uma escala de 1 (discordo totalmente) até 7 (concordo totalmente) para a avaliação de um conjunto de itens relacionados ao controle, à clareza dos objetivos, à autonomia, ao feedback, à imersão, ao conhecimento e ao desafio.

Figura 1. Avaliação dos usuários utilizando EGameFlow (versão desktop)

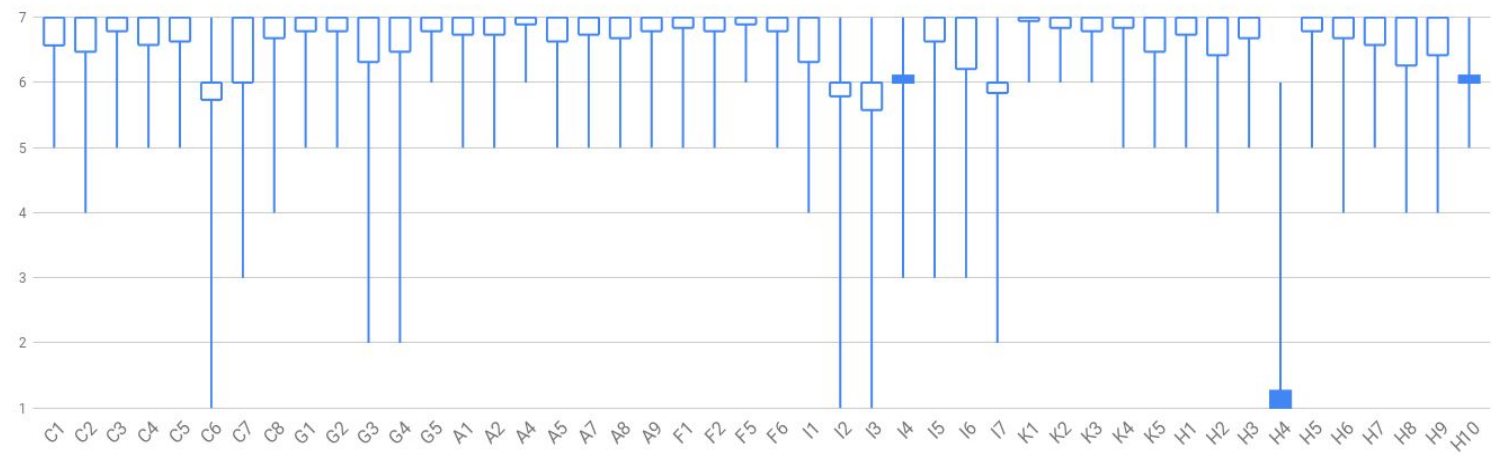

Fonte:[REIS, 2020]

Figura 2. Avaliação dos usuários utilizando EGameFlow (versão mobile)

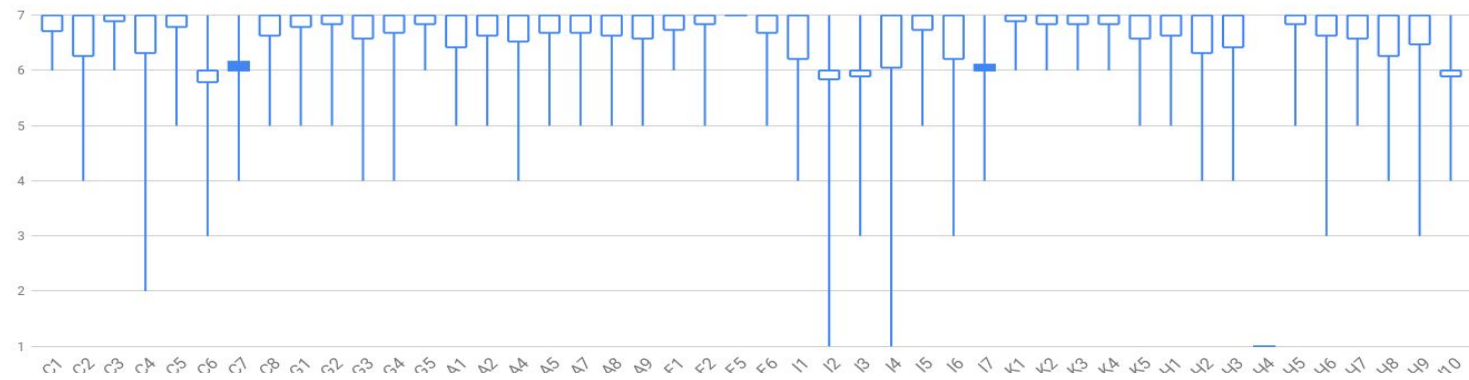

Fonte:[REIS, 2020]

As Figuras 1 e 2 mostram as notas mínimas e máximas (representadas pelo início e final das linhas verticais), além das médias e medianas para cada item do EGameFlow atribuídas pelos 19 participantes, respectivamente nas versões desktop e mobile. As médias e medianas estão representadas respectivamente pelas bases inferior e superior dos retângulos não preenchidos, e o contrário no caso dos retângulos preenchidos (indicando que a mediana é menor que a média). A análise dos gráficos 
mostra que as médias e medianas de quase todos os itens ficaram acima de 6 e apenas uma ficou abaixo de 4, indicando satisfação dos usuários com o jogo.

Os itens com pior avaliação estão relacionados ao desafio e à imersão no jogo. Todos os usuários testaram uma instância do jogo com o mesmo conteúdo (questões relacionadas às capitais dos estados brasileiros), independentemente da idade e escolaridade. Considerando que o jogo possui apenas uma fase e a mecânica é bastante simples, as sensações de desafio e imersão estavam diretamente relacionadas ao conhecimento do participante sobre o tema. Assim, alguns julgaram o jogo muito fácil e outros se sentiram mais desafiados e imersos. Esse problema tende a ser solucionado no uso real, já que o seu conteúdo das questões poderá ser customizado pelo professor por meio da plataforma REMAR. Algumas questões relacionadas à imersão e à concentração apresentaram grandes variações entre as versões desktop e mobile. Isso ocorreu porque os usuários cegos e de baixa visão se mostraram mais concentrados e imersos utilizando a versão desktop. Os dados consolidados para cada grupo estão em [REIS 2020].

Em síntese, podemos concluir que: (1) as avaliações por especialistas confirmaram a adequação do jogo Forca Inclusiva às recomendações do EduGameAccess-M, ou seja, foram bem interpretadas e bem empregadas pela equipe de desenvolvimento do jogo; (2) os testes de jogabilidade com usuários dos 4 grupos mostraram que o jogo Forca Inclusiva promoveu boas experiências de jogo com os usuários dos 4 grupos, sinalizando a efetividade do conjunto EduGameAccess- $M$ no apoio ao design de um jogo educacional multidispositivo que inclui pessoas com deficiência visual.

\section{Considerações Finais}

Neste trabalho é apresentado brevemente o processo de design, implementação e avaliação do jogo Forca Inclusiva, que foi teve como base o conjunto de recomendações $E d u$ GameAccess- $M$. Os resultados iniciais obtidos indicam que o conjunto proposto contribuiu efetivamente para o desenvolvimento de um jogo educacional web inclusivo, capaz de engajar pessoas com e sem deficiência visual, em suas versões desktop e mobile. O jogo produzido está disponível como recurso educacional aberto na plataforma REMAR e pode ser utilizado para a criação de instâncias de jogo com conteúdo customizado diretamente por professores sem conhecimento em programação.

\section{Agradecimentos}

O presente trabalho foi realizado com apoio da Coordenação de Aperfeiçoamento de Pessoal de Nível Superior - Brasil (CAPES) - Código de Financiamento 001.

\section{Referências}

ARCHAMBAULT D, OSSMANN R, GAUDY T, MIESENBERGER K (2007) Computer games and visually impaired people. Upgrade 8(2):43-53.

BALAN O, MOLDOVEANU A, MOLDOVEANU F. (2015). Navigational audio games: An effective approach toward improving spatial contextual learning for blind people. International Journal on Disability and Human Development, 14(2), 109-118.

BEDER DM, OTSUKA JL. (2019). A platform for customization and publication of open educational games, In: Proceedings of XXX Simpósio Brasileiro de Informática na Educação 
IX Congresso Brasileiro de Informática na Educação (CBIE 2020)

Anais do XXXI Simpósio Brasileiro de Informática na Educação (SBIE 2020)

(SBIE 2019). https://doi.org/10.5753/cbie.sbie.2019.902.

DESURVIRE H, WIBERG C (2009) Game Usability Heuristics (PLAY) for Evaluating and Designing Better Games: The Next Iteration. In: OCSC'09 Proceedings of the 3d International Conference on Online Communities and Social Computing: Held as Part of HCI International 2009, USA, pp 557-566.

FIZEK S, WOLETZ JD, BEKSA J (2015) Playing with sound and gesture in digital audio games. From prototype design to evaluation. In: Weisbecker A, Burmester M, Schmidt A (eds) Mensch \& Computer Workshopband.

FONTOURA JUNIOR PHF. (2018). Recomendações para o Desenvolvimento de Jogos Educacionais Integrando Aspectos de Acessibilidade, Jogabilidade e Educacionais. Dissertação de Mestrado. Programa de Pós Graduação em Computação - UFSCar.

FU FL, SU RC, YU SC (2009) Egameflow: A scale to measure learners' enjoyment of e-learning games. Computers \& Education, Elsevier, v. 52, n. 1, p. 101-112, 2009.

FRIBERG J, GÄRDENFORS D (2004) Audio Games: New Perspectives on Game Audio. In: Proceedings of the 2004 ACM SIGCHI International Conference on Advances in Computer Entertainment Technology, ACM, USA, pp. 148-154.

GAG (2019) Game Accessibility Guidelines. Available at: http://gameaccessibilityguidelines.com , accessed on August 24, 2019.

GEE JP (2007) Good Video Games + Good Learning: Collected Essays on Video Games, Learning, and Literacy. Peter Lang Publishing, USA Hartley S (2011) World report on disability (who).

GLINERT, EM. The human controller: usability and accessibility in video game interfaces. Tese (Doutorado) - Massachusetts Institute of Technology, 2008.

GRABSKI A, TONI T, ZIGRAND T, WELLER R, ZACHMANN G. Kinaptic- techniques and insights for creating competitive accessible $3 \mathrm{~d}$ games for sighted and visually impaired users. In: IEEE. Haptics Symposium (HAPTICS), 2016 IEEE. [S.1.], 2016. p. 325-331.

GRAMMENOS, D.; SAVIDIS, A.; STEPHANIDIS, C. Designing universally accessible games. Computers in Entertainment (CIE), ACM, v. 7, n. 1, p. 8, 2009.

IBGE (2010) CENSO DEMOGRÁFICO 2010. Available at: http://biblioteca.ibge.gov.br/visualizacao/periodicos/94/cd_2010_religiao_deficiencia.pdf , accessed on October 17, 2019.

IGDA (2019) Game Access SIG.Available at: https://igda- gasig.org/ about-_game-_accessibility/guidelines/visual/accessed on August 24, 2019.

KORHONEN, H. Evaluating playability of mobile games with the expert review method.Tampere University Press, 2016.

NETO LV, FONTOURA JUNIOR PHF, BORDINI RA, OTSUKA JL, BEDER DM, Details on the Design and Evaluation Process of an Educational Game Considering Issues for Visually Impaired People Inclusion. Educational Technology \& Society, 22 (3), 4-18.

ÖSTBLAD A, ENGSTRÖM H, BRUSK J, BACKLUND P. AND WILHELMSSON U(2014). Inclusive game design: audio interface in a graphical adventure game. In Proceedings of the 9th Audio Mostly: A Conference on Interaction With Sound (AM '14). Association for Computing Machinery, New York, NY, USA, Article 29, 1-8. DOI:https://doi.org/10.1145/2636879.2636909.

REIS,APS(2020). Jogos Educacionais Inclusivos para diferentes dispositivos. Dissertação de Mestrado. Programa de Pós Graduação em Computação - UFSCar.

WORLD HEALTH ORGANIZATION (2011). World report on disability 2011. Available at: https://www.who.int/disabilities/world_report/2011/report/en/.

YUAN B, FOLMER E, HARRIS JR FC (2011) Game accessibility: a survey. Universal Access in the Information Society 10(1):81-100. 